\title{
An Adaptive Companding Scheme for Peak-to-average Power Ratio Reduction in OFDM Systems
}

\author{
Sana Mazahir ${ }^{1}$, Shahzad Amin Sheikh ${ }^{2}$ \\ ${ }^{1}$ Department of Electrical Engineering, College of Electrical and Mechanical Engineering, \\ National University of Sciences and Technology, Rawalpindi, Pakistan \\ [e-mail: sana75@ee.ceme.edu.pk] \\ ${ }^{2}$ Department of Electrical Engineering, College of Electrical and Mechanical Engineering, \\ National University of Sciences and Technology, Rawalpindi, Pakistan \\ [e-mail: sheikh.shahzadamin@gmail.com] \\ *Corresponding author: Sana Mazahir
}

Received May 5, 2015; revised July 8, 2015; revised August 3, 2015; accepted August 12, 2015; published December 31, 2015

\begin{abstract}
Orthogonal frequency division multiplexing (OFDM) signals suffer from the problem of high peak-to-average power ratio (PAPR), which complicates the design of analog front-end of the system. Companding is a well-known PAPR reduction technique that involves transforming signal amplitudes using a deterministic function. OFDM signal amplitude, on average, is Rayleigh distributed but the distribution can vary significantly from symbol to symbol, especially when constellation size increases. In this paper, a new adaptive companding scheme is proposed along with its design methodology aiming at optimizing the compander performance by accommodating this variation in its design. This is achieved by designing compander parameters separately for statistically dissimilar symbols in OFDM waveform and making the compander select from these parameters, during run-time, according to the features of input symbols.
\end{abstract}

Keywords: OFDM, PAPR reduction, companding transform, adaptive companding 


\section{Introduction}

Orthogonal frequency division multiplexing (OFDM) has been extensively deployed in high speed wireless communication systems, including broadband internet access systems and broadcast systems. It offers the advantages of high spectral efficiency, immunity to multipath fading and power efficiency. However, due to the addition of multiple carriers with random amplitudes and phases, OFDM signal envelope has large fluctuations. This increases the dynamic range of the signal amplitude, which causes performance degradation resulting from non-linear operation of high power amplifier (HPA) at the transmitter.

Peak-to-average power ratio (PAPR) is the most common metric employed for quantifying envelope fluctuations in OFDM signals. Several techniques have been proposed to reduce the PAPR of OFDM signals [1-2]. Some of them are probabilistic schemes, such as selective mapping (SLM), partial transmit sequences (PTS), tone injection (TI), tone reservation (TR) and active constellation extension (ACE). PAPR can also be reduced by deterministic operations, like iterative clipping and filtering (ICF), peak windowing and companding.

In the above mentioned techniques, PAPR can be reduced at the expense of one or two of the following undesirable modifications in the system: increased average power, high computational complexity, out-of-band radiation, degraded error performance and data rate loss [1-2]. A flexible and efficient scheme would offer the opportunity to exploit most of the tolerances in the system, so that it would have more operating regions and hence more adaptability to the changes in input and channel conditions. PTS, SLM and TR achieve PAPR reduction by increasing computational complexity and introducing data rate loss, while TI and ACE involve increase in both computational complexity and average power as trade-offs to the same end. These techniques, however, do not have the amenability to acquire any advantage either from possibly inherent system's resilience to error or from favorable channel conditions. On the other hand, clipping and companding are low in complexity but cause significant signal distortion, that results in degraded error performance and spectral regrowth. Moreover, they are deterministic operations on random input that do not take into account the statistical variations of the input; rather they are designed on the basis of average or long-term signal statistics. If the variations in signal statistics are significant, especially when larger signal constellations are used, the output signal characteristics may not conform well to the preset specifications. Some instances of these companders are piecewise linear transforms in [3-4] and non-linear transforms in [5-9]. For all of these companders, PAPR reduction performance evaluation has been presented only for QPSK or 4-QAM based signals, in which all symbols have equal power. So the effects of aforementioned variations are not as readily discernible from the complementary cumulative distribution functions (CCDFs) of PAPR as they would have been in case of higher order QAM constellations.

In this paper, a new adaptive companding scheme is proposed along with its design methodology. The main idea is to make the compander select its parameters from a predetermined set according to the statistical feature(s) of the input OFDM symbol. The selection of parameters is done in such a way that the change in average power and overall amplitude distribution from symbol to symbol can be accounted for in the companding transformation. This is demonstrated to have greater control over the output signal characteristics and enhanced PAPR reduction capability, while keeping the mean signal distortion same as in case of the corresponding deterministic or fixed compander. Having greater control over the signal characteristics is desirable because it can ensure higher 
efficiency of non-linear components in the transmitter, including HPA and digital-to-analog converter (DAC). Furthermore, unlike fixed companders, the design framework used in this scheme makes it realizable to exploit tolerance for data rate loss and surplus computational, memory and power resources in the system for PAPR reduction. Hence the proposed adaptive companding scheme has the ability to expand the trade-space over bandwidth efficiency, required transmission power and computational complexity in addition to PAPR reduction performance, error performance and out-of-band radiation levels, thereby integrating the flexibilities of probabilistic and deterministic schemes. This means that system's adaptability to input and channel conditions will be enhanced because now it can have more possible regions of operation as compared to those viable for systems with other PAPR reduction schemes.

The remainder of this article has been organized as follows. In Section 2, a typical OFDM system model is described. General design concept and formal definitions for the proposed scheme are given in Section 3. In Section 4, the design methodology is proposed. Simulation results are presented and discussed in Section 5. Finally, conclusion is drawn in Section 6.

\section{System Model}

Fig. 1 shows the block diagram of a typical OFDM system with the proposed adaptive companding scheme. The oversampled, discrete-time, complex baseband equivalent of the transmitted OFDM signal is given as follows:

$$
x_{n}=\frac{1}{\sqrt{N L}} \sum_{k=0}^{N L-1} X_{k} e^{\frac{j 2 \pi k n}{N L}}, 0 \leq n \leq N L-1
$$

where $X_{k}$ comes from input symbol vector $\boldsymbol{X}=[X_{0}, X_{1}, X_{2}, \ldots, X_{\frac{N}{2}-1}, \underbrace{0, \ldots, 0}_{N(L-1)}, X_{\frac{N}{2}}, \ldots$, $\left.X_{N-1}\right]$ in which each symbol is modulated using QAM constellation. $N$ is the number of subcarriers including data carriers, pilot carriers and null carriers and $L$ is the upsampling factor.

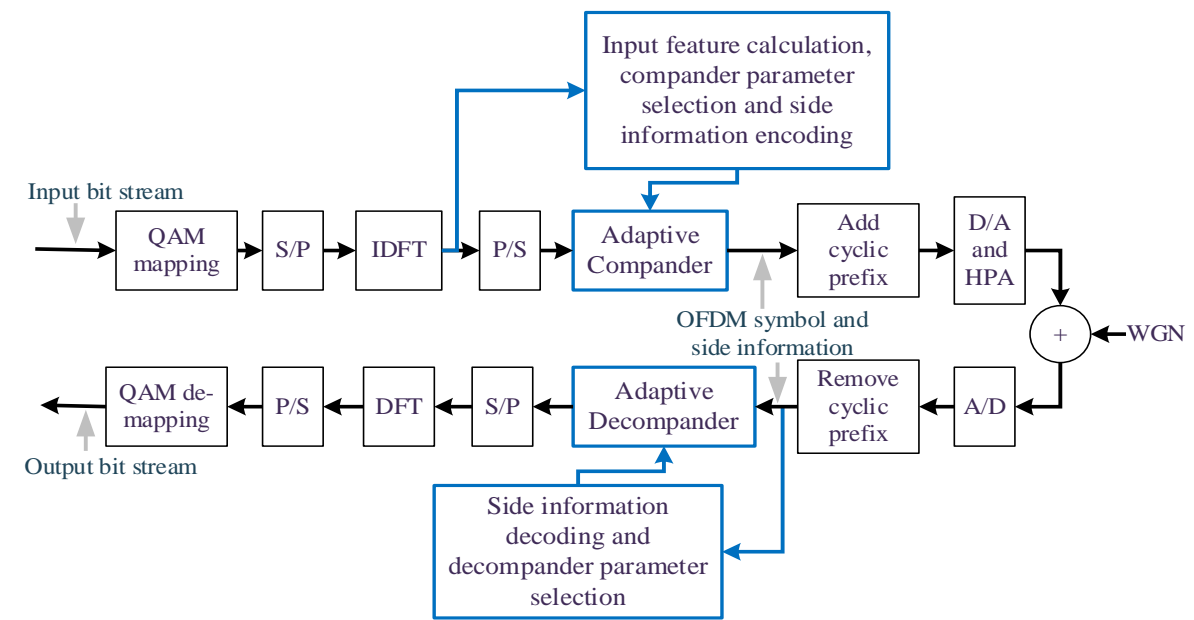

Fig. 1. OFDM system with the proposed adaptive companding scheme 
With the assumption that each $X_{k}$ can independently have any value from a given QAM constellation with equal probability, $x_{n}$ becomes a random process generated by weighted addition of independent and identically distributed (IID) random variables. When $N$ is large, $x_{n}$ can be approximated as a complex Gaussian random process using the central limit theorem approximation. The amplitude of a complex Gaussian random process has Rayleigh distribution. The cumulative distribution function (CDF) and probability density function (PDF) of amplitude of $x_{n}$ are given by

$$
\begin{aligned}
F_{A}(x)=1-e^{-\frac{x^{2}}{\sigma_{x}^{2}}}, & x \geq 0 \\
f_{A}(x)=\frac{2 x}{\sigma_{x}^{2}} e^{-\frac{x^{2}}{\sigma_{x}^{2}}}, & x \geq 0
\end{aligned}
$$

where $A$ is the symbol used for random variable associated with $\left|x_{n}\right|$ throughout this text. $\sigma_{x}^{2}$ is the variance of $x_{n}$ given by $E\left[\left|x_{n}\right|^{2}\right]=E\left[\left|X_{k}\right|^{2}\right]=\frac{1}{N L}\left(\varepsilon_{x} N_{\text {data }}+\varepsilon_{p} N_{\text {pilot }}\right)$, where $N_{\text {data }}$ and $N_{\text {pilot }}$ are the number of data and pilot subcarriers respectively and $\varepsilon_{x}$ and $\varepsilon_{p}$ are average energies of data and pilot symbols respectively. For an M-ary square QAM constellation, $\varepsilon_{x}=\frac{d^{2}}{6}(M-1)$, where $d$ is the minimum distance between constellation points.

The PAPR of the complex baseband equivalent $x_{n}$ of the transmitted OFDM signal is defined as follows.

$$
\operatorname{PAPR}(d B)=10 \log _{10}\left(\frac{\max _{0 \leq n \leq N L-1}\left|x_{n}\right|^{2}}{\frac{1}{N L} \sum_{n=0}^{N L-1}\left|x_{n}\right|^{2}}\right)
$$

\section{General Concept and Formalization of Adaptive Companding}

In general, companders are deterministic functions designed to modify OFDM signal amplitude after modulation operation, while assuming that the amplitude of the signal is a Rayleigh random process. However, the Rayleigh process approximation applies for large data sets, which, in this case, is the complete OFDM waveform comprising of sufficiently large number of symbols. This means that the companders designed using Rayleigh PDF are, in effect, designed for an average OFDM symbol. The deviation of amplitude statistics of individual OFDM symbols from the Rayleigh distribution is not taken into account. For a given number of subcarriers, this deviation becomes more pronounced as the constellation size increases and is manifested in the compander output as increased variances of signal attributes, like average signal power and PAPR, around their desired or preset values. The variances imply that the compander under-performs for some symbols and over-performs for others. 


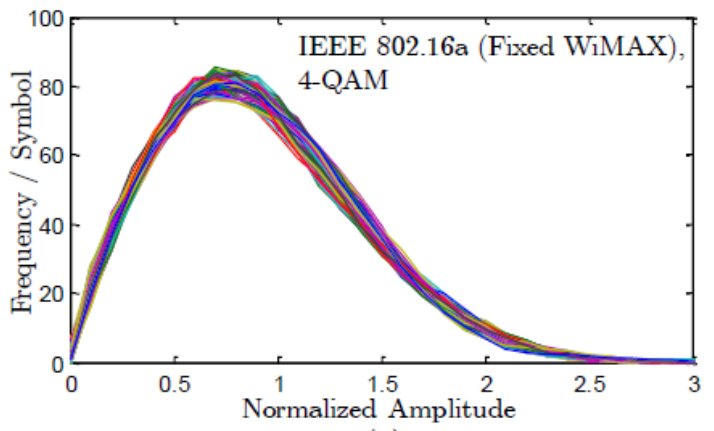

(a)

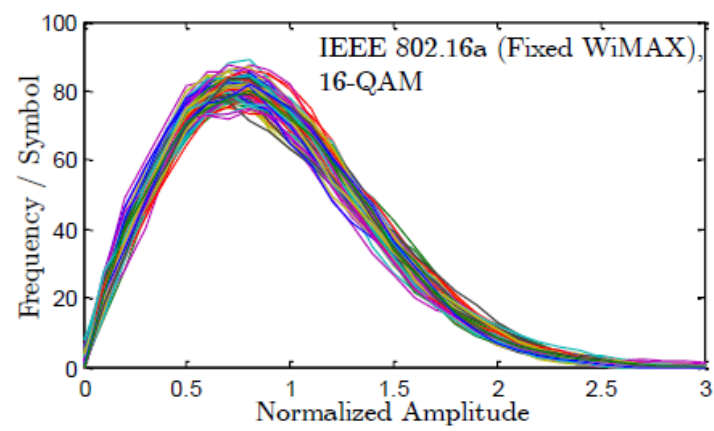

(b)

Fig. 2. Histogram envelopes of amplitude of 50 realizations of OFDM symbols with (a) 4-QAM and (b) 16-QAM modulation

Fig. 2 shows amplitude distributions of simulated OFDM symbols. The variations of amplitude statistics can be clearly seen. This variation is larger in case of 16-QAM than that for 4-QAM. In 4-QAM and PSK modulation schemes with same number of subcarriers, average power of all OFDM symbols remains same while in larger QAM constellations, average power also varies in addition to overall amplitude distribution. This means that the output of companders designed to preserve average power may show more significant aberrations from expected behavior when larger QAM constellations are used for modulation.

The idea of proposed adaptive companding scheme is based on the premise that by introducing input data dependencies in compander parameter evaluation or selection, the deviation of output signal characteristics from design specifications can be reduced, so that the compander performance would be closer to optimal; instead of over-performing or under-performing, it will be able to perform as required. Smaller variance of signal power along with reduced PAPR will facilitate smaller required input back-off (IBO) and hence improved power efficiency of HPA. Moreover, the mean signal distortion remains same as in case of the corresponding fixed compander.

The design approach for the proposed scheme is elaborated as follows. The OFDM waveform (ignoring the cyclic prefix) can be thought of as a set of symbols such that the amplitude statistics of some symbols resemble more closely than others. This set is divided into subsets in such a way that within each subset, the variation of amplitude statistics affecting the compander performance is smaller as compared to that in the complete set. The compander parameters are then calculated for each of these subsets separately and independently. Since the amplitude statistics are now incorporated in the compander design, so the output of the compander can be expected to exhibit better concordance with the original design objectives.

Let $S$ denote the random variable associated with the statistical measure of signal amplitude, on the basis of which the OFDM waveform is divided into $J$ subsets. Definition of the statistical measure $S$ depends upon the form of compander function, such that change in $S$ is causative of change in compander output from its desired value. We define $J$ disjoint, consecutive intervals of $S$ :

$$
\begin{aligned}
& {\left[s_{0}, s_{1}\right],\left(s_{1}, s_{2}\right],\left(s_{2}, s_{3}\right], \ldots,\left(s_{J-1}, s_{J}\right],} \\
& \text { where } s_{0}<s_{1}<s_{2}<\cdots<s_{J-1}<s_{J}
\end{aligned}
$$

$s_{0}=\min (S), s_{J}=\max (S)$ and $s_{1}, s_{2}, \ldots, s_{J-1}$ are selected such that the variance of $S$ is 
equal within all intervals given above,

$$
\begin{aligned}
\operatorname{Var}\left(S \mid s_{0} \leq\right. & \left.S \leq s_{1}\right)=\operatorname{Var}\left(S \mid s_{1}<S \leq s_{2}\right)=\cdots=\operatorname{Var}\left(S \mid s_{J-1}<S \leq s_{J}\right) \\
& <\operatorname{Var}(S)
\end{aligned}
$$

Since $S$ is such a statistical measure that the compander function and its output are directly affected by its value, the conditions on variances are imposed in order to ensure that compander performance will be similar in all subsets.

Now $j^{\text {th }}$ subset of OFDM waveform is defined as:

$$
\left\{\boldsymbol{x}_{\boldsymbol{n}} \mid S \text { of }\left|\boldsymbol{x}_{\boldsymbol{n}}\right| \in\left(s_{j-1}, s_{j}\right]\right\}, \text { for } j=1,2, \ldots, J
$$

where $\boldsymbol{x}_{\boldsymbol{n}}=\left[x_{0}, x_{1}, \ldots, x_{N L-1}\right]$ and $\left|\boldsymbol{x}_{\boldsymbol{n}}\right|=\left[\left|x_{0}\right|,\left|x_{1}\right|, \ldots,\left|x_{N L-1}\right|\right]$.

The probability distributions of signal amplitude for $j^{\text {th }}$ subset are evaluated by modifying the original distributions, given in Eq. (2), to conditional distributions:

$$
\begin{gathered}
F_{A}\left(x \mid s_{j-1}<S \leq s_{j}\right)=\operatorname{Pr}\left[A \leq x \mid s_{j-1}<S \leq s_{j}\right] \\
f_{A}\left(x \mid s_{j-1}<S \leq s_{j}\right)=\frac{d}{d x} F_{A}\left(x \mid s_{j-1}<S \leq s_{j}\right)
\end{gathered}
$$

Since modified distributions, given in Eq. (7), will now be used to describe amplitude statistics in respective subsets instead of Rayleigh distribution and the compander parameters for each subset will be calculated using these distributions, so the compander parameters will be optimized according to the value of $S$ for symbols in each subset.

Now the compander is designed for each of the $J$ subsets separately using the conditional distributions given in Eq. (7), so that we have a set of $J$ compander parameter values. Companding transform on signal amplitudes in an OFDM symbol can be expressed as follows:

$$
T\left(\left|\boldsymbol{x}_{\boldsymbol{n}}\right|\right)=\left\{\begin{array}{cc}
T_{1}\left(\left|\boldsymbol{x}_{\boldsymbol{n}}\right|\right), & S \text { of }\left|\boldsymbol{x}_{\boldsymbol{n}}\right| \in\left[s_{0}, s_{1}\right] \\
T_{2}\left(\left|\boldsymbol{x}_{\boldsymbol{n}}\right|\right), & S \text { of }\left|\boldsymbol{x}_{\boldsymbol{n}}\right| \in\left(s_{1}, s_{2}\right] \\
& \vdots \\
T_{J}\left(\left|\boldsymbol{x}_{\boldsymbol{n}}\right|\right), & S \text { of }\left|\boldsymbol{x}_{\boldsymbol{n}}\right| \in\left(S_{J-1}, s_{J}\right]
\end{array}\right.
$$

If $D$ is a measure of companding distortion for a certain companding function and all of the $J$ companders have similar mathematical form, design specifications and constraints, then, by virtue of the law of total expectation, the over-all mean companding distortion remains same as for the fixed compander.

$$
E[D]=\sum_{j=1}^{J} \underbrace{E\left[D \mid s_{j-1}<S \leq s_{j}\right]}_{\begin{array}{c}
\text { mean distortion in } \\
j^{\text {th }} \text { subset }
\end{array}} \underbrace{\operatorname{Pr}\left[s_{j-1}<S \leq s_{j}\right]}_{\begin{array}{c}
\text { fraction of waveform in } \\
j^{\text {th }} \text { subset }
\end{array}}
$$

If error performance and spectral characteristics are determined by $D$, then it means that the BER and out-of-band radiation levels will remain same in fixed and adaptive companders, irrespective of the number of subsets $J$.

The adaptive compander will operate by first calculating the statistic $S$ for the input OFDM symbol, then selecting its parameter values from the compander parameter set and then the selected function will transform the signal amplitudes. In order to correctly recover the signal at the receiver end, compander parameters will need to be communicated to the receiver, so that same parameters are used by the decompander. Since number of possible parameter values or compander profiles, as given in Eq. (8), is $J$, so the minimum number of 
bits needed to uniquely encode the parameter values will be $\left\lceil\log _{2} J\right\rceil$, where $\lceil$.$\rceil denotes$ ceiling function. Hence $\left\lceil\log _{2} \mathrm{~J}\right\rceil$ bits of side information will need to be transmitted with each symbol. The decompander will determine its parameters from the same set by examining this side information.

\section{Design Methodology}

In this section, the design methodology for adaptive compander is developed using the piecewise linear companding transform recently proposed in [3].

\subsection{Companding Transform}

The piecewise linear companding transform, presented in [3], and its inverse are defined using three parameters: clipping level $\alpha_{c}$, inflexion point $\alpha_{i}$ and slope $k$. The transform is applied on the amplitude of the signal while phase remains unchanged.

$$
\begin{aligned}
& T(x)=\left\{\begin{aligned}
x, & x \leq \alpha_{i} \\
k x+(1-k) \alpha_{c}, & \alpha_{i}<x \leq \alpha_{c} \\
\alpha_{c}, & x>\alpha_{c}
\end{aligned}\right. \\
& T^{-1}(x)=\left\{\begin{aligned}
x, & x \leq \alpha_{i} \\
\alpha_{i}, & \alpha_{i}<x \leq k \alpha_{i}+(1-k) \alpha_{c} \\
\frac{x-(1-k) \alpha_{c}}{k}, & k \alpha_{i}+(1-k) \alpha_{c}<x \leq \alpha_{c} \\
\alpha_{c}, & x>\alpha_{c}
\end{aligned}\right.
\end{aligned}
$$

$\alpha_{c}$ is determined by setting a preset value of PAPR such that $\alpha_{c}=\sigma_{x} 10^{P A P R_{\text {preset }} / 20}$ and $\alpha_{i}$ and $k$ are determined according to following design objectives and constraints: average power remains constant and companding distortion is minimized. $D=(T(x)-x)^{2}$ is used as the measure of distortion. Mean distortion is given by $\sigma_{c}^{2}=E\left[(T(x)-x)^{2}\right]$ and is shown to be related to receiver's signal-to-noise ratio (SNR) in [3].

\subsection{Problem Formulation in Adaptive Compander Design Framework}

The parameters $\alpha_{c}, k$ and $\alpha_{i}$ in Eq. (10) are now selected from a set according to the value of statistical feature $S . \alpha_{c}$ depends upon preset PAPR specification and average signal power, so it will remain constant for all OFDM symbols in case of PSK and 4-QAM. However, it may be varied for other QAM constellations.

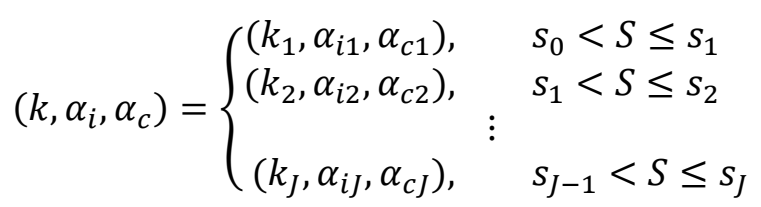

For $j^{\text {th }}$ interval of $S,\left(k_{j}, \alpha_{i j}, \alpha_{c j}\right)$ are calculated using the modified form of design equations given in [3].

Clipping level is defined for symbols in each subset separately. Since $P A P R_{\text {preset }}=$ $10 \log _{10} \alpha_{c j}^{2} / \sigma_{x j}^{2}$,

$$
\alpha_{c j}=\sigma_{x j} 10 \frac{P A P R_{\text {preset }}}{20}
$$


Companding distortion is defined for each of the $J$ subsets individually instead of for the whole waveform as in [3]:

$$
\begin{gathered}
\sigma_{c j}^{2}=\int_{0}^{\infty}\left(T_{j}(x)-x\right)^{2} f_{A}\left(x \mid s_{j-1}<S \leq s_{j}\right) d x \\
\sigma_{c j}^{2}=\left(k_{j}-1\right)^{2} I_{2}\left(\alpha_{i j}, \alpha_{c j}\right)+I_{2}\left(\alpha_{c j}, \infty\right)-2\left(1-k_{j}\right)^{2} \alpha_{c j} I_{1}\left(\alpha_{i j}, \alpha_{c j}\right) \\
+\left(1-k_{j}\right)^{2} \alpha_{c j}^{2} I_{0}\left(\alpha_{i j}, \alpha_{c j}\right)+\alpha_{c j}^{2} I_{0}\left(\alpha_{c j}, \infty\right)-2 \alpha_{c j} I_{1}\left(\alpha_{c j}, \infty\right)
\end{gathered}
$$

where $T_{j}(x)=\left.T(x)\right|_{\left(k, \alpha_{i}, \alpha_{c}\right)=\left(k_{j}, \alpha_{i j}, \alpha_{c j}\right)}$ and $I_{0}, I_{1}, I_{2}$ are integrals given by $I_{n}\left(x_{i}, x_{f}\right)=$ $\int_{x_{i}}^{x_{f}} x^{n} f_{A}\left(x \mid s_{j-1}<S \leq s_{j}\right) d x$ for given $S$ and $j$. Estimator for the integrals is given Subsection 4.5. Likewise, the constraint on average power is also defined for each subset individually instead of assuming same distribution for all symbols as in [3]:

$$
\begin{gathered}
\sigma_{x j}^{2}=\int_{0}^{\infty} x^{2} f_{A}\left(x \mid s_{j-1}<S \leq s_{j}\right) d x=\int_{0}^{\infty} T_{j}(x)^{2} f_{A}\left(x \mid s_{j-1}<S \leq s_{j}\right) d x \\
{\left[I_{2}\left(\alpha_{i j}, \alpha_{c j}\right)+\alpha_{c j}^{2} I_{0}\left(\alpha_{i j}, \alpha_{c j}\right)-2 \alpha_{c j} I_{1}\left(\alpha_{i j}, \alpha_{c j}\right)\right] k_{j}^{2}} \\
+\left[-2 \alpha_{c j}^{2} I_{0}\left(\alpha_{i j}, \alpha_{c j}\right)+2 \alpha_{c j} I_{1}\left(\alpha_{i j}, \alpha_{c j}\right)\right] k_{j} \\
+\left[\alpha_{c j}^{2} I_{0}\left(\alpha_{i j}, \infty\right)+I_{2}\left(0, \alpha_{i j}\right)-\sigma_{x j}^{2}\right]=0
\end{gathered}
$$

Following the approach in [3], Eq. (14a) has been expressed as a quadratic equation with respect to $k_{j}$ in Eq. (14b).

Now $\left(k_{j}, \alpha_{i j}, \alpha_{c j}\right)$ are such that the companding distortion $\sigma_{c j}^{2}$ is minimized while keeping average power $\sigma_{x j}^{2}$ unchanged for a given value of $P A P R_{\text {preset }}$. Eqs. (12), (13) and (14) represent $J$ sets of design equations, each of which will be solved separately and independently, for $j=1,2, \ldots, J$, using the optimization algorithm in [3] and the estimations given in Sub-section 4.5 .

In addition to optimizing the compander performance for the given objectives and constraints, the incorporation of new parameters in the problem framework provides additional degrees of freedom in specifying the objectives and constraints as compared to the fixed companders. Consequently, more types of trade-offs are possible. In fixed companders, PAPR reduction can be improved only by increasing signal distortion but in this case, it is possible to realize a number of operating points for the system with different levels of reduced PAPR at same signal distortion level, while the trade-offs for PAPR reduction are shifted to other, possibly more tolerant, system attributes. This enhances the system in terms of flexibility, efficiency and adaptability to input and channel conditions.

\subsection{Selection of Statistical Feature}

The statistical feature $S$ used to select compander parameter should be such a measure on signal amplitude that the compander operation is directly affected by it, which means that this feature is being used in the calculation of compander parameters.

For constellations with changing symbol energy, like 16-QAM, fixed compander assumes that average power $\sigma_{x}^{2}$ remains constant and equal to the long-term average power 
of the OFDM waveform. However, when considering individual symbols, $\sigma_{x}^{2}$ is not equal in all symbols. Also, average power is the feature that affects the compander performance because it is being used in the evaluation of its parameters, as shown in Eqs. (12) and (14). So the average symbol power can be used as the feature to adapt.

$$
S=\text { Power }_{\text {avg }}=\frac{1}{N L} \sum_{n=0}^{N L-1}\left|x_{n}\right|^{2}=\frac{1}{N L} \sum_{n=0}^{N L-1}\left|X_{k}\right|^{2}
$$

So now instead of using same $\sigma_{x j}^{2}=\sigma_{x}^{2}$ in all symbols, $\sigma_{x j}^{2}$ can be set equal to the average symbol power in $j^{\text {th }}$ subset.

$$
\sigma_{x j}^{2}=\int_{0}^{\infty} x^{2} f_{A}\left(x \mid s_{j-1}<S \leq s_{j}\right) d x=I_{2}(0, \infty)
$$

However, in PSK and 4-QAM modulation, average power remains constant. So the input feature must be based on how the overall amplitude distribution affects the compander performance. In the compander function under consideration, peak power is reduced by clipping operation and the power deficiency created due to clipping is compensated by expanding the smaller amplitudes. Since clipping level is set by Eq. (12), it will remain constant because average power $\sigma_{x j}^{2}$ remains constant for all $j$. But the exact amount of power deficiency due to clipping changes from symbol to symbol and hence the requirement of power compensation also changes. The change in amount of power deficiency is due to the fact that the number of samples being clipped and their amplitudes are not exactly similar in all symbols. If compander parameters are evaluated while incorporating this change, its output will be closer to optimal, which makes it pertinent to choose $S$ as the difference between the powers of original and clipped signals. Hence the statistic $S$ is defined as follows:

$$
S=\text { Power }_{\text {original }}-\text { Power }_{\text {clipped }}=\sum_{A \in\left\{\left|x_{n}\right|\right\}, A>\alpha_{C}}\left(A^{2}-\alpha_{c}^{2}\right)
$$

where $\left\{\left|x_{n}\right|\right\}=\left\{\left|x_{0}\right|,\left|x_{1}\right|, \ldots,\left|x_{N L-1}\right|\right\}$.

\subsection{Partitioning of OFDM Waveform}

Intervals on $S$ are calculated according to Eqs. (4) and (5) using simulated data comprising of 200000 realizations. Simulation parameters are same as given in Section 5. If $J$ is a power of two, then the intervals of $S$ are found by successively bisecting maximum variance intervals using Algorithm 1. At each iteration, the current interval with maximum variance of $S$ is divided into two, such that the two new intervals are approximately equal in variance of $S$.

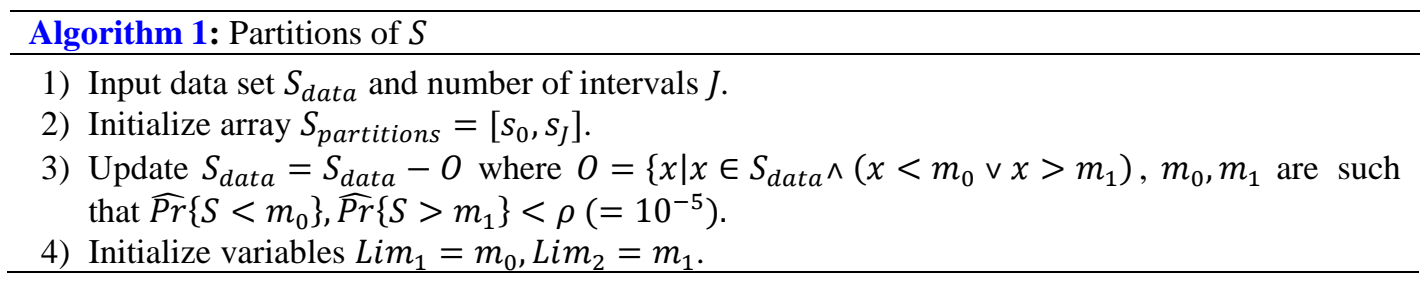


5) Find $s_{\text {new }} \in\left(\operatorname{Lim}_{1}, \operatorname{Lim}_{2}\right)$ such that for

dataset $_{1}=\left\{x \mid x \in S_{\text {data }} \wedge \operatorname{Lim}_{1}<x \leq s_{\text {new }}\right\}$,

dataset $_{2}=\left\{x \mid x \in S_{\text {data }} \wedge S_{\text {new }}<x \leq \operatorname{Lim}_{2}\right\}$,

$\mid \operatorname{Var}\left(\right.$ dataset $\left._{1}\right)-\operatorname{Var}\left(\right.$ dataset $\left._{2}\right) \mid$ is minimized.

6) Append $s_{\text {new }}$ to array $S_{\text {partitions }}$ and sort $S_{\text {partitions }}$ in ascending order.

7) Find $\quad i \in\left\{1,2, \ldots\right.$, length $\left.\left(S_{\text {partitions }}\right)-1\right\}$ such that for

dataset $_{i}=\left\{x \mid x \in S_{\text {data }} \wedge S_{\text {partitions }}(i)<x \leq S_{\text {partitions }}(i+1)\right\}, \quad \operatorname{Var}\left(\right.$ dataset $\left._{i}\right) \quad$ is maximum.

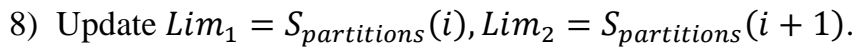

9) If length $\left(S_{\text {partitions }}\right)<J+1$, repeat steps 5-8; otherwise output $S_{\text {partitions }}$ and terminate.

\subsection{Conditional Probability Distributions}

In order to evaluate the integrals in Eqs. (13), (14) and (16), the conditional probability distributions are required. For each of the $J$ subsets found according to Eq. (6), conditional CDFs are estimated using simulated data as follows:

$$
\hat{F}_{A}\left(x \mid s_{j-1}<S \leq s_{j}\right)=\frac{\text { No. of samples in } j^{\text {th }} \text { subset with amplitude } \leq x}{\text { Total no. of samples in } j^{\text {th }} \text { subset }}
$$

where $x$ varies in small increments from zero to maximum amplitude present in the complete data set and $s_{0}, s_{1}, \ldots, s_{J}$ are outputs of Algorithm 1. PDFs can be estimated using numerical gradient of respective CDFs. Fig. 3 shows the estimated probability distributions for $J=4$ for 4-QAM modulation using the feature $S$, given in Eq. (17).

Since the empirical CDF curves have smaller approximation error, they are used to estimate the integrals in Eqs. (13), (14) and (16) as follows:

$$
\begin{gathered}
I_{n}\left(x_{i}, x_{f}\right)=\int_{x_{i}}^{x_{f}} x^{n} f_{A}\left(x \mid s_{j-1}<S \leq s_{j}\right) d x=\int_{x_{i}}^{x_{f}} x^{n} \frac{d}{d x} F_{A}\left(x \mid s_{j-1}<S \leq s_{j}\right) d x \\
=\left.x^{n} F_{A}\left(x \mid s_{j-1}<S \leq s_{j}\right)\right|_{x_{i}} ^{x_{f}}-\int_{x_{i}}^{x_{f}} n x^{n-1} F_{A}\left(x \mid s_{j-1}<S \leq s_{j}\right) d x \\
\approx \underbrace{x_{f}^{n} \hat{F}_{A}\left(x_{f} \mid s_{j-1}<S \leq s_{j}\right)-x_{i}^{n} \hat{F}_{A}\left(x_{i} \mid s_{j-1}<S \leq s_{j}\right)}_{\begin{array}{c}
\text { Numerical Integration } \\
\text { using Trapezoidal Rule }
\end{array}} \\
-\underbrace{\int_{x_{f}}^{x_{f}} n x^{n-1} \hat{F}_{A}\left(x \mid s_{j-1}<S \leq s_{j}\right) d x}_{x_{i}}
\end{gathered}
$$

where $x_{i}$ and $x_{f}$ are limits of integration, $n=0,1,2$ and $j=1,2, \ldots, J$. Limits at infinity in Eqs. (13), (14) and (16) have been replaced by maximum amplitude for which $\hat{F}_{A}($.$) is$ available. Now the $J$ sets of design equations can be solved for $\left(k_{j}, \alpha_{i j}, \alpha_{c j}\right)$ using the optimization algorithm in [3] along with the integral estimator in Eq. (19). Fig. 4 shows the compander functions designed using the conditional distributions shown in Fig. 3. 


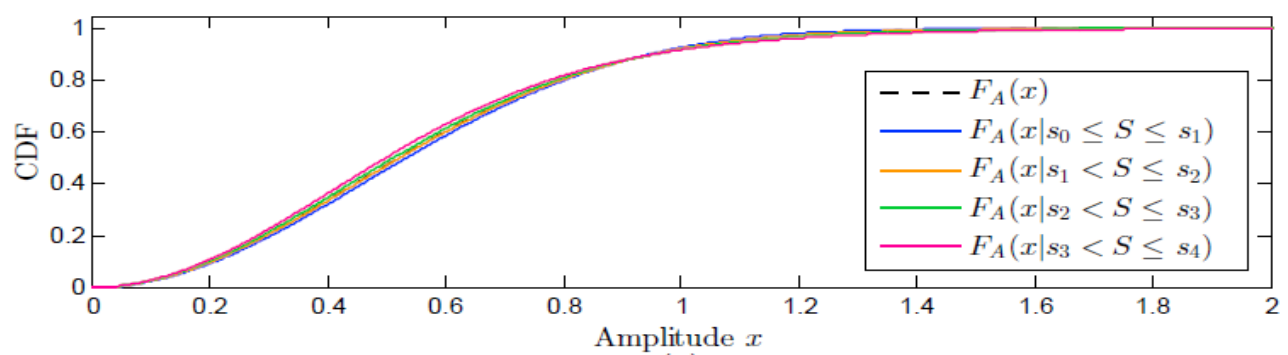

(a)

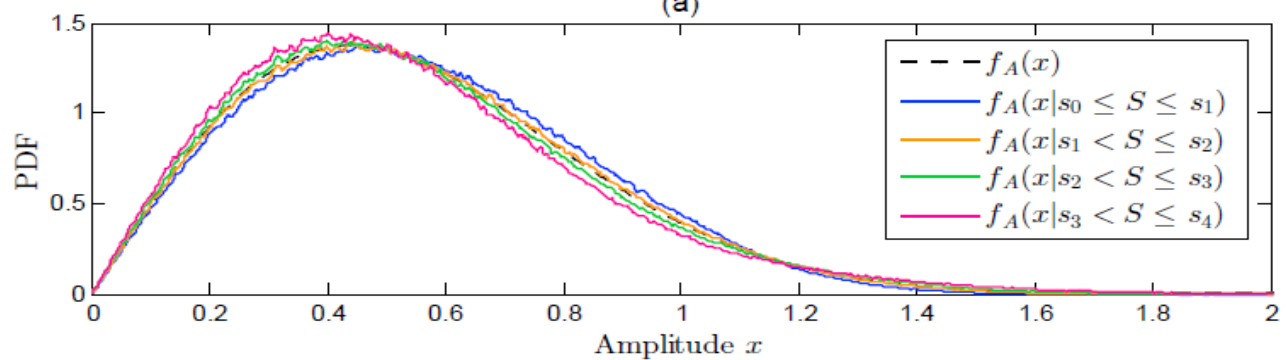

(b)

Fig. 3. Estimated conditional probability distributions. $J=4, S=$ Power $_{\text {original }}-$ Power $_{\text {clipped }}$, $s_{0}, s_{1}, s_{2}, s_{3}, s_{4}$ are outputs of Algorithm 1. OFDM symbols are based on 4-QAM constellation.

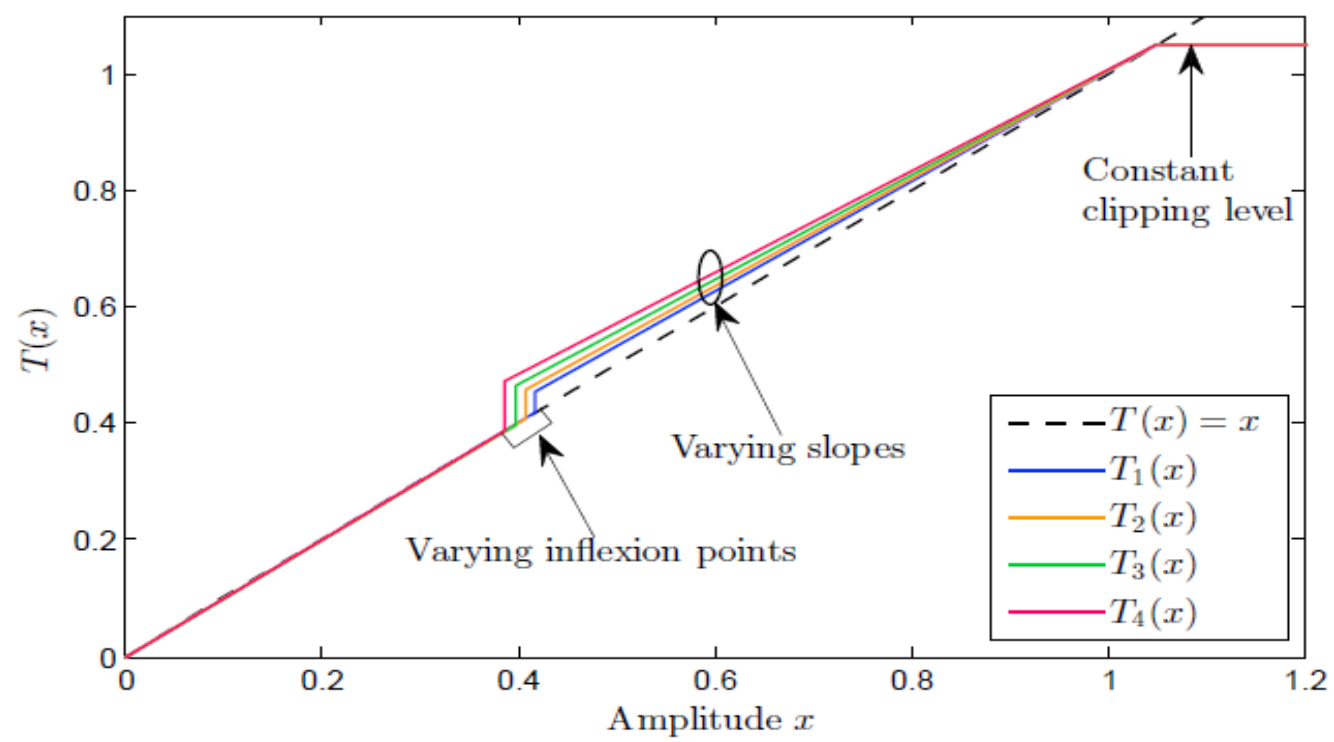

Fig. 4. Companding transform profiles, designed by using the estimated distributions, shown in Fig. 3, in Eqs. (12), (13) and (14). For constant clipping level $\alpha_{c}$, slopes $k_{j}$, inflexion points $\alpha_{i j}$ are separately calculated for $j=1,2,3,4$ to get $T_{1}(x), T_{2}(x), T_{3}(x), T_{4}(x)$, respectively.

\section{Performance Evaluation}

The performance of the proposed companding scheme, in terms of PAPR reduction, BER, power spectral density (PSD), computational complexity and amount of required side information, is evaluated using simulated OFDM symbols. The OFDM symbols are simulated according to physical layer specifications given in IEEE 802.16 standard used in Fixed Worldwide Interoperability for Microwave Access (WiMAX). Total number of subcarriers $N$ is 256 which includes 192 data carriers, 8 pilot carriers and 56 null carriers 
(guard band and DC). The oversampling factor $L$ is 4. 4-QAM and 16-QAM are used as modulation schemes.

\subsection{With 4-QAM based OFDM}

Figs. 5-7 show performance evaluation for OFDM system with 4-QAM modulation. In Fig. 5, PAPR reduction performance evaluation is presented for $P A P R_{\text {preset }}=$ $4 \mathrm{~dB}, 4.5 \mathrm{~dB}$ and $5 \mathrm{~dB}$. For each case, fixed and adaptive versions of the compander are simulated. Statistical feature $S$ is same as given in Eq. (17). It can be clearly seen that as the number of subsets $J$ increases from 2 to 32, realized PAPR values come closer to their respective preset values. At $C C D F=10^{-4}$, improvement of approximately $0.25 \mathrm{~dB}$ for $J=32$ is observed as compared to fixed compander.

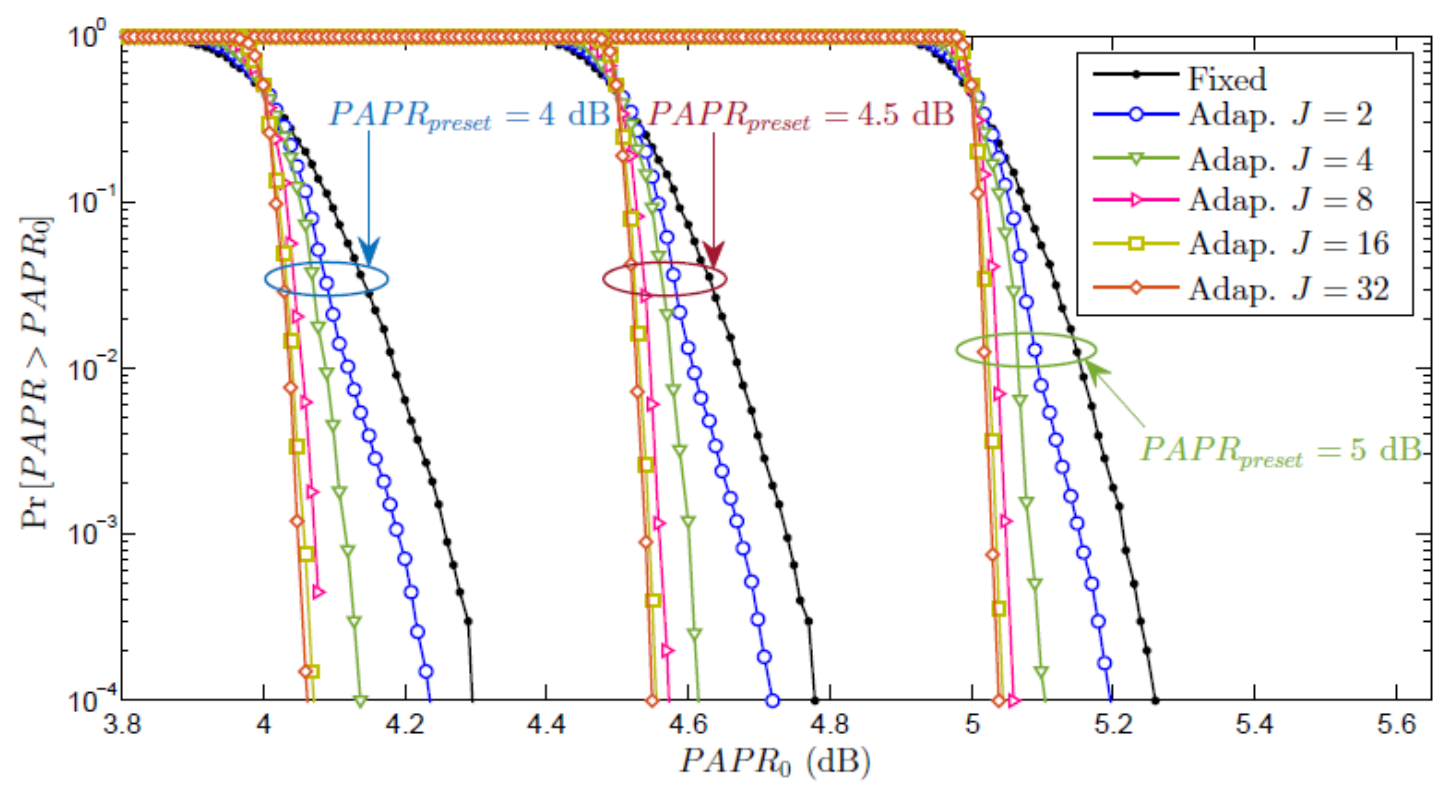

Fig. 5. CCDFs of PAPR of OFDM signals transformed by fixed and adaptive companders. OFDM signals are based on 4-QAM constellation.

BER performance, over AWGN channel, for all the cases is shown in Fig. 6. For each value of $P A P R_{\text {preset }}$, error performance remains similar for fixed and adaptive companders. Six coinciding BER curves, one for fixed compander and five for adaptive companders, with $J=2,4,8,16$ and 32 , are obtained in each case.

In Fig. 7, average PSDs, calculated using the periodogram estimates, are shown to be coincident for fixed compander and the best case (with respect to PAPR reduction performance) of adaptive $(J=32$ ) for all the three values of preset PAPR values. The observations of BER and PSD show that the average companding distortion remains same in fixed and adaptive companders, as predicted by Eq. (9). 


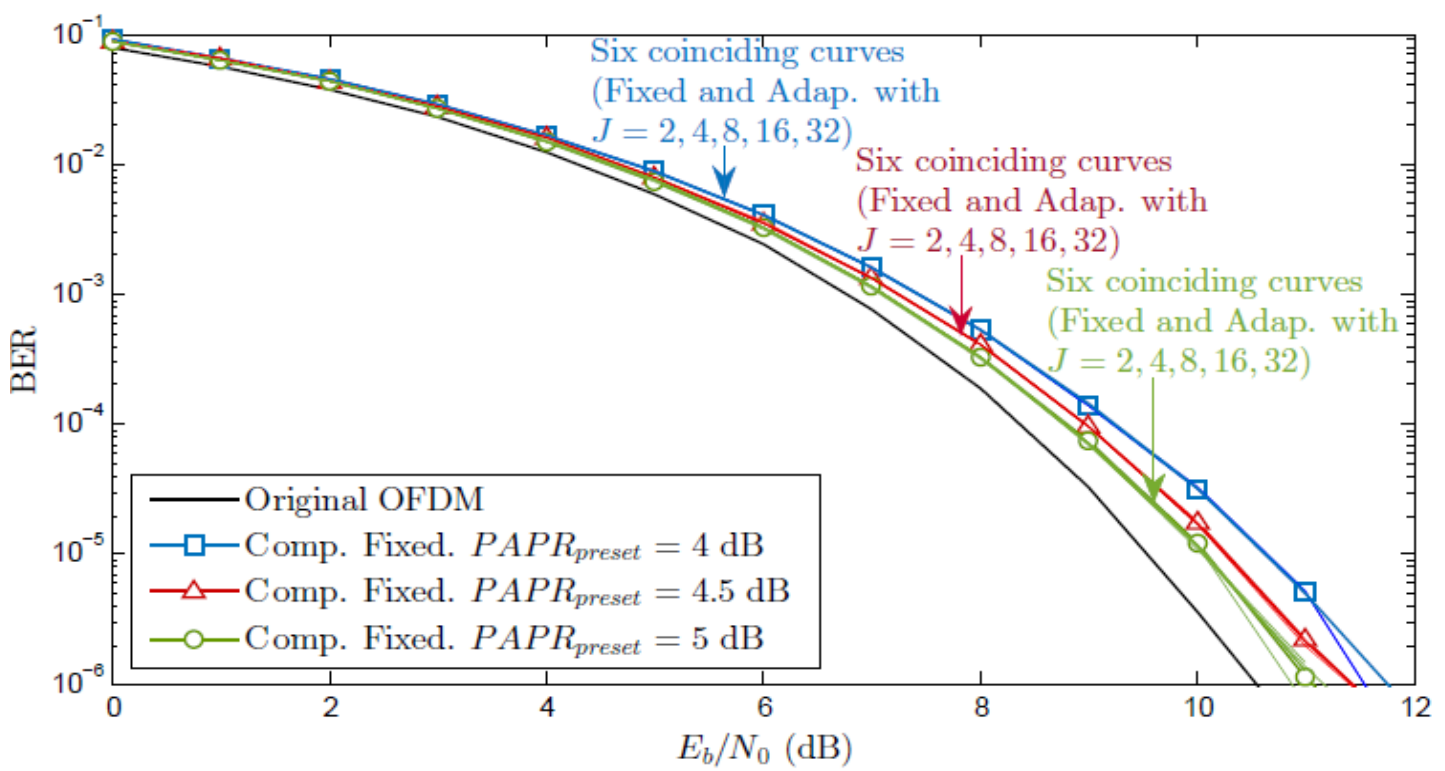

Fig. 6. BER performance of original OFDM signal and OFDM signal transformed with fixed and adaptive companders (for $J=2,4,8,16,32$ ) over AWGN channel using 4-QAM modulation.

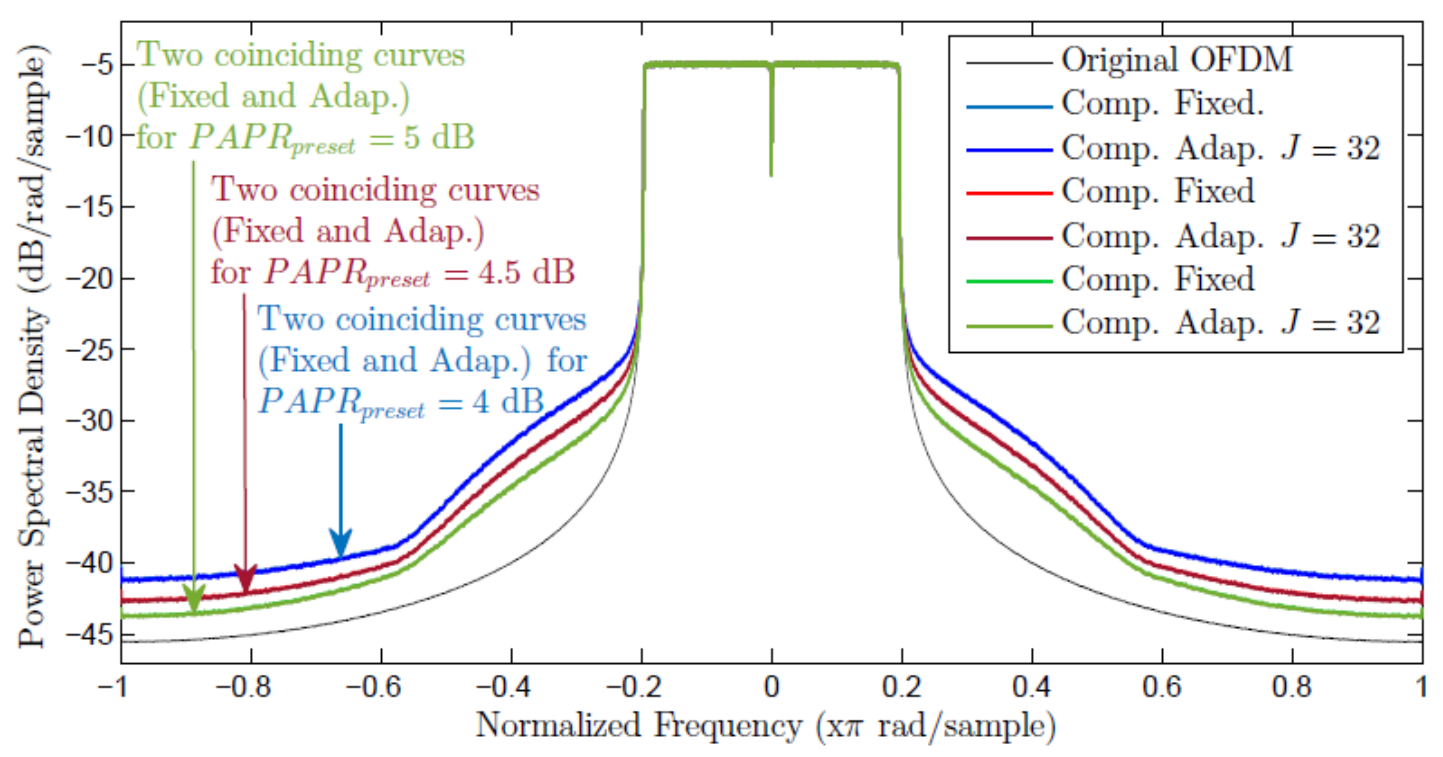

Fig. 7. PSDs of original OFDM signal and OFDM signal transformed with fixed and adaptive companders. OFDM signals are based on 4-QAM constellation.

\subsection{With 16-QAM based OFDM}

Figs. 8-12 show performance evaluation with 16-QAM constellation. It can be seen in Fig. 8 that the CCDF curve for fixed compander with $P A P R_{\text {preset }}=4.5 \mathrm{~dB}$, using 16QAM, shows significant performance degradation (approximately $0.34 \mathrm{~dB}$ at $C C D F=10^{-4}$ ) as compared to the fixed compander with same $P A P R_{\text {preset }}$, using 4-QAM modulation, shown in Fig. 5. This is due to the fact that unlike 4-QAM constellation, the average power 
per symbol for OFDM signals based on 16-QAM constellation has non-zero variance, which is not accounted for in the fixed compander design equations. So in addition to overall variation in amplitude distribution, the changing symbol power becomes an additional factor contributing to the deviation of realized PAPR from its preset value.

As discussed in Sub-section 4.2, the incorporation of new variables in the design framework makes it realizable to obtain many different operating conditions for the system using the same companding transform. This type of flexibility is important in PAPR reduction schemes because it allows manageable trade-offs in many different situations and the same technique can meet performance constraints in many applications. It has been shown in [2] that none of the existing PAPR reduction techniques can provide good tradeoffs in all situations. The proposed adaptive companding scheme has the capability that it allows the distribution of trade-offs for PAPR reduction among error performance (BER), spectral spreading (PSD), computational complexity, data rate loss and average power, which is not possible in any conventional PAPR reduction scheme [1-2].

In order to illustrate the all-encompassing trade-space and enhanced flexibility in obtaining various operating conditions for the system with the proposed design framework, companders are designed and evaluated in five different scenarios:

i. $S=$ Power $_{\text {avg }}, \alpha_{c j}=\alpha_{c}=\sigma_{x} 10^{P A P R_{\text {preset }} / 20}, \sigma_{x j}^{2}=\sigma_{x}^{2}$ in Eq. (14), where $\sigma_{x}^{2}$ is the long-term average power of the OFDM waveform, given in Section 2. These conditions aim at providing such a power scaling that all symbols can be transmitted at same power level $\sigma_{x}^{2}$. Since average power is same, clipping level will also be constant according to Eq. (12). Figs. 8-10 show evaluation for this case with $J=2,4,8,16,32$.

ii. $S=$ Power $_{a v g}, \alpha_{c j}=\sigma_{x j} 10^{P A P R_{\text {preset }} / 20} . \sigma_{x j}^{2}$, in Eqs. (12) and (14), is calculated using Eq. (16). In this case, compander is designed to maintain the average power of individual symbols. Figs. 8, 11 and 12 show results for this case with $J=16$. Additional power scaling will be needed at the transmitter if it is required to maintain constant transmission power, but BER will be improved due to the elimination of unnecessary clipping, as observed in Fig. 11.

iii. $S=$ Power $_{\text {avg }}$. For every $j=1,2, \ldots, \frac{J}{2}, \alpha_{c j}=\sigma_{x} 10^{P A P R_{\text {preset }} / 20}$ and $\sigma_{x j}^{2}=\sigma_{x}^{2}$. For every $j=\frac{J}{2}+1, \frac{J}{2}+2, \ldots, J, \alpha_{c j}=\sigma_{x j} 10^{P A P R_{\text {preset }} / 20}$ and $\sigma_{x j}^{2}$, in Eqs. (12) and (14), is calculated using Eq. (16). In this case, the power of those symbols in which $\sigma_{x j}^{2} \leq \sigma_{x}^{2}$ is increased to overall average power $\sigma_{x}^{2}$ and the symbols in which $\sigma_{x j}^{2}>\sigma_{x}^{2}$, power is kept unchanged. This is done to increase the SNR for low power symbols by a small increase in average power resulting in overall reduction in BER, as shown in Fig. 11. Increase in average power results in increase in both in-band and out-of-band power, which means that the side-lobe level is increased, as shown in Fig. 12. Results for this case are shown in Figs. 8, 11 and 12 with $J=16$.

iv. Two features are used. $S_{1}=$ Power $_{\text {avg }}, S_{2}=$ Power $_{\text {original }}-$ Power $_{\text {clipped }}$. OFDM waveform is divided into $J_{1}$ subsets based on $S_{1}$. Each of the $J_{1}$ subsets is further divided into $J_{2}$ subsets based on $S_{2} . \alpha_{c j_{1}}=\sigma_{x j_{1}} 10^{P A P R_{\text {preset }} / 20}$, for $j_{1}=1,2, \ldots, J_{1}$. Figs. 8, 11 and 12 show results for this case with $J_{1}=8, J_{2}=4$ and $J_{1}=8, J_{2}=8$.

v. $S=$ Power $_{\text {avg }} \cdot \alpha_{c j}=\sigma_{x j} 10^{P A P R_{\text {preset }} / 20}$ and $\sigma_{x j}^{2}=\sigma_{x j}^{2}+\Delta \sigma_{x j}^{2}$. Overall average power is increased to raise SNR and hence reduce BER. Also increase in average power 
results in raised side-lobe level as shown in Fig. 12, in addition to increased in-band power. Figs. 8, 11 and 12 show results for this case with $J=16$.

In all five cases, the changes in amplitude distribution will be incorporated by using the specified $S$ in conditional distributions in Eqs. (13) and (14). In scenario (iv), conditions on $S_{1}$ and $S_{2}$ are simultaneously imposed. The conditional distributions are estimated as described in Sub-section 4.5.

In Fig. 8, PAPR reduction performance comparison of fixed and adaptive companders for $P A P R_{\text {preset }}=4.5 \mathrm{~dB}$ is presented. The results of CCDF of PAPR obtained for scenarios (i), (ii), (iii) and (v) were found to be approximately similar. Only those for (i) are shown in Fig. 8. This shows that PAPR reduction performance depends on $J$ or in turn the extent of similarity of feature $S$ within a subset. Improvement of approximately $0.31 \mathrm{~dB}$ at $C C D F=$ $10^{-4}$ is achieved with $J=16$ and $J=32$ as compared to the fixed compander.

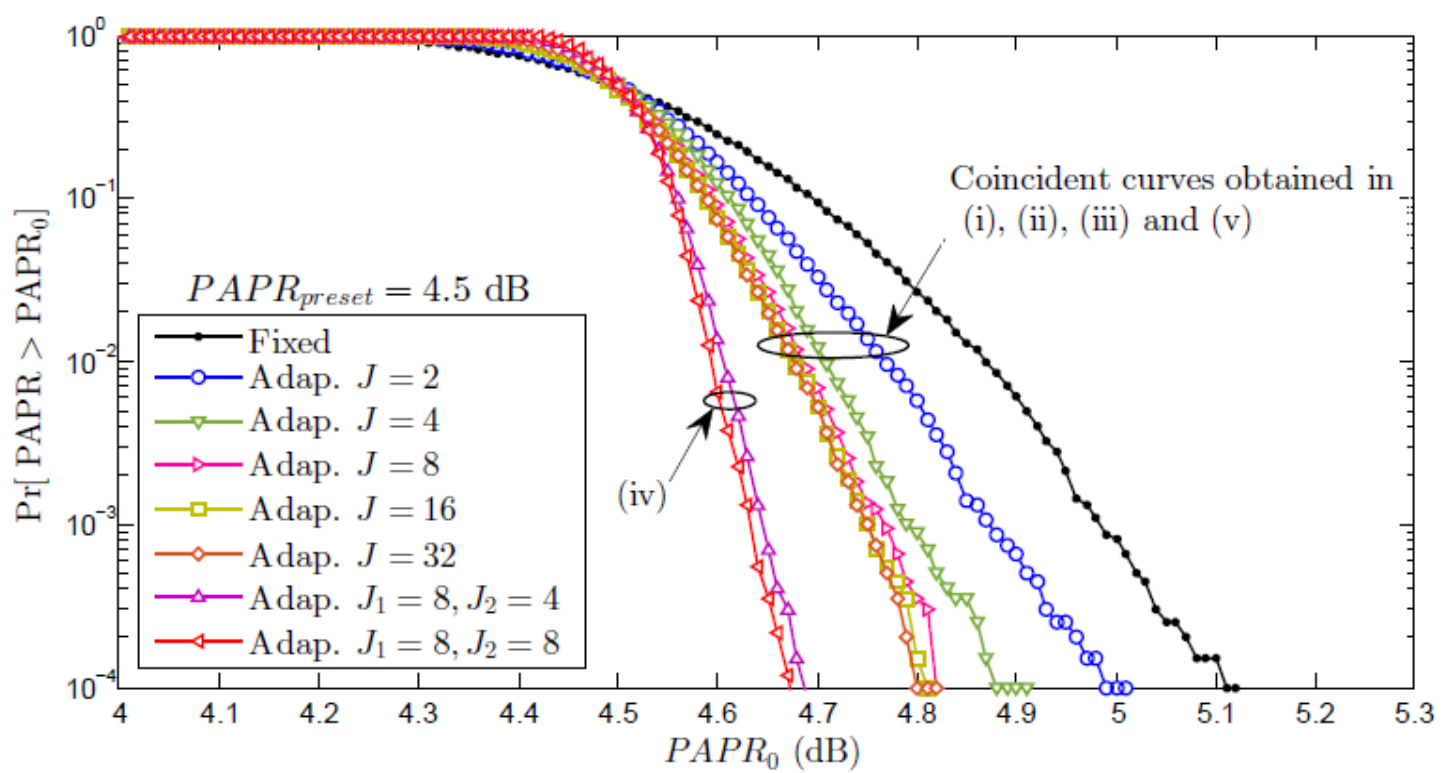

Fig. 8. CCDFs of PAPR of OFDM signals transformed by fixed and adaptive companders. OFDM signals are based on 16-QAM constellation.

It can be seen that as $J$ increases in (i), (ii), (iii) and (v), the CCDF approaches the CCDF of 4-QAM with fixed compander in Fig. 5. This means that since within each subset, variation of average power is very small, the amplitude statistics within the subsets approach those of PSK or 4-QAM. Hence the best PAPR reduction performance achievable, using average power in Eq. (15) as feature, is the performance of 4-QAM with fixed compander. In order to further reduce PAPR, a second feature $S_{2}$ is required to incorporate the overall distribution variation, as done in case of 4-QAM. This has been done according to scenario (iv). Further improvement of $0.12 \mathrm{~dB}$ is obtained with $J_{1}=8, J_{2}=8$, as shown in Fig. 8.

Figs. 9 and 10 show BER and PSD, respectively, for companders designed according to conditions in scenario (i). Performance is similar to that of the fixed one for $J=$ $2,4,8,16$ and 32. BER and PSD remain unchanged irrespective of $J$, as predicted by Eq. (9). 


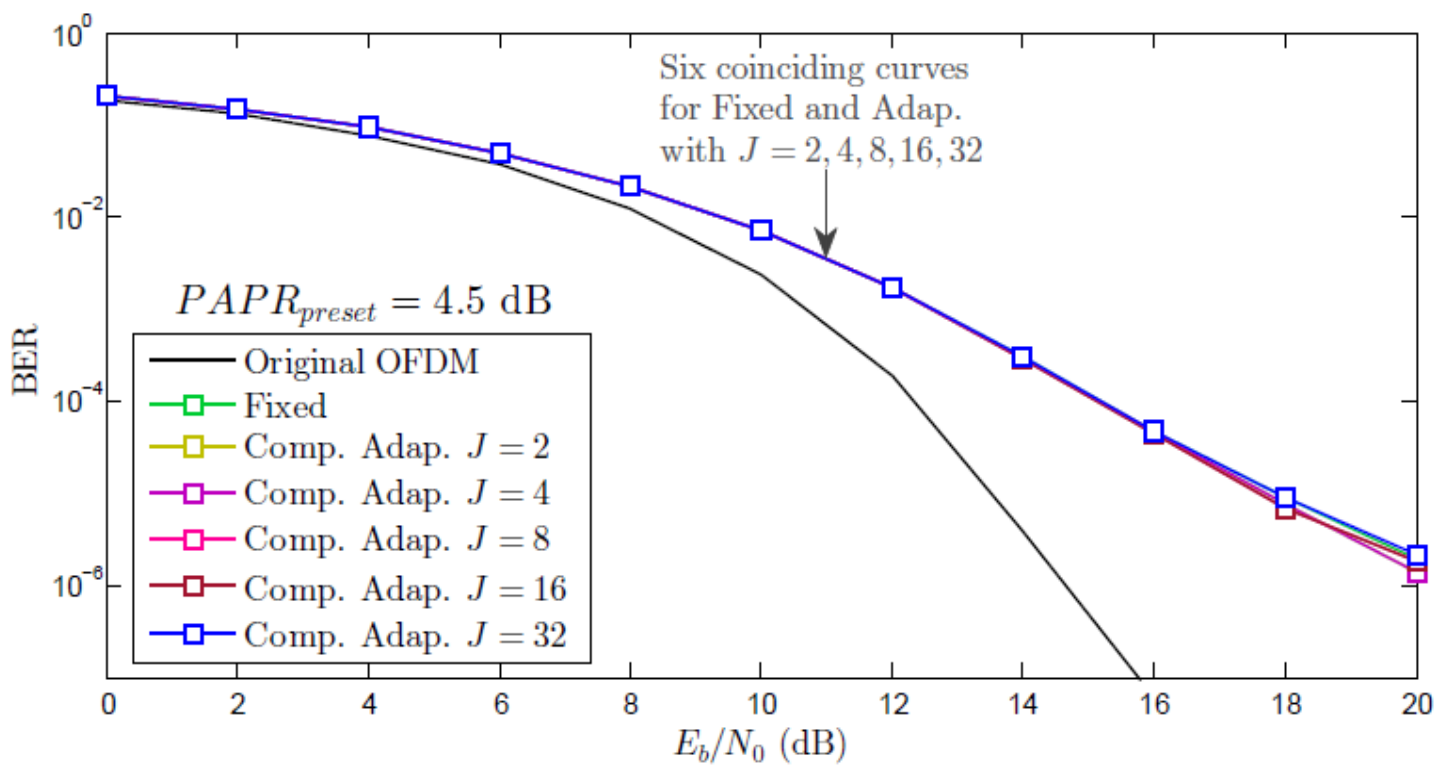

Fig. 9. BER performance of original OFDM signal and OFDM signal transformed with fixed and adaptive companders in scenario (i), over AWGN channel, using 16-QAM modulation.

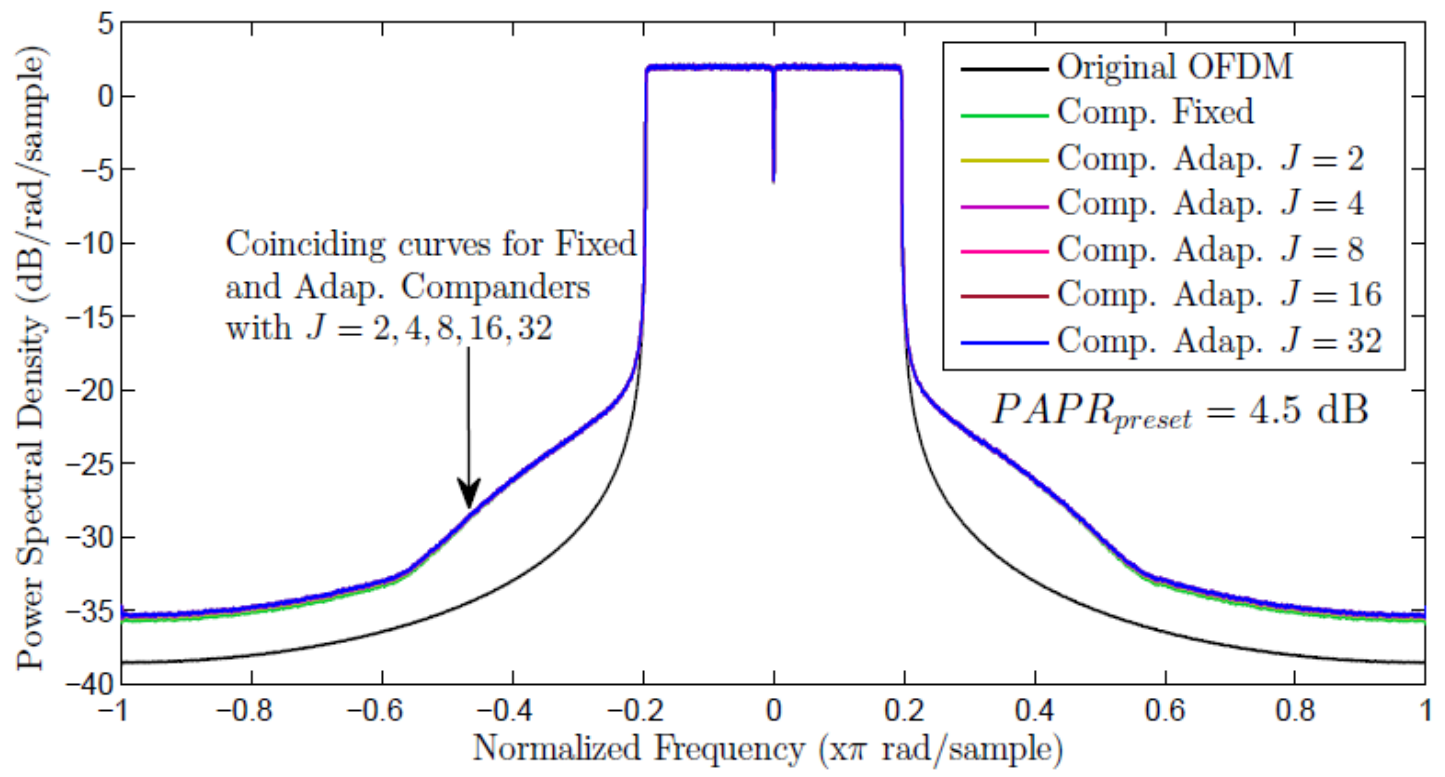

Fig. 10. PSDs of original OFDM signal and OFDM signal transformed with fixed and adaptive companders in scenario (i). OFDM signals are based on 16-QAM constellation.

Figs. 11 and 12 show BER and PSD, respectively, for companders design in scenarios (ii), (iii) and (v) for the best case (with respect to PAPR reduction/side information trade-off), i.e., $J=16$. BER and PSD for scenario (iv) are shown for $J_{1}=8, J_{2}=8$, which is found to be the best case with respect to PAPR reduction in this scenario.

In scenarios (ii) and (iv), PSD remains unchanged while BER at high SNR is slightly reduced, which may be explained by the fact that unnecessary clipping is being avoided in both cases. So the performance floor at high SNR, due to clipping noise, has been lowered.

In scenarios (iii) and (v), BER is reduced both due to overall increase in signal power 
and elimination of unnecessary clipping operation. In case of PSD, both in-band and out-ofband energy is increased due to the increase in overall average power.

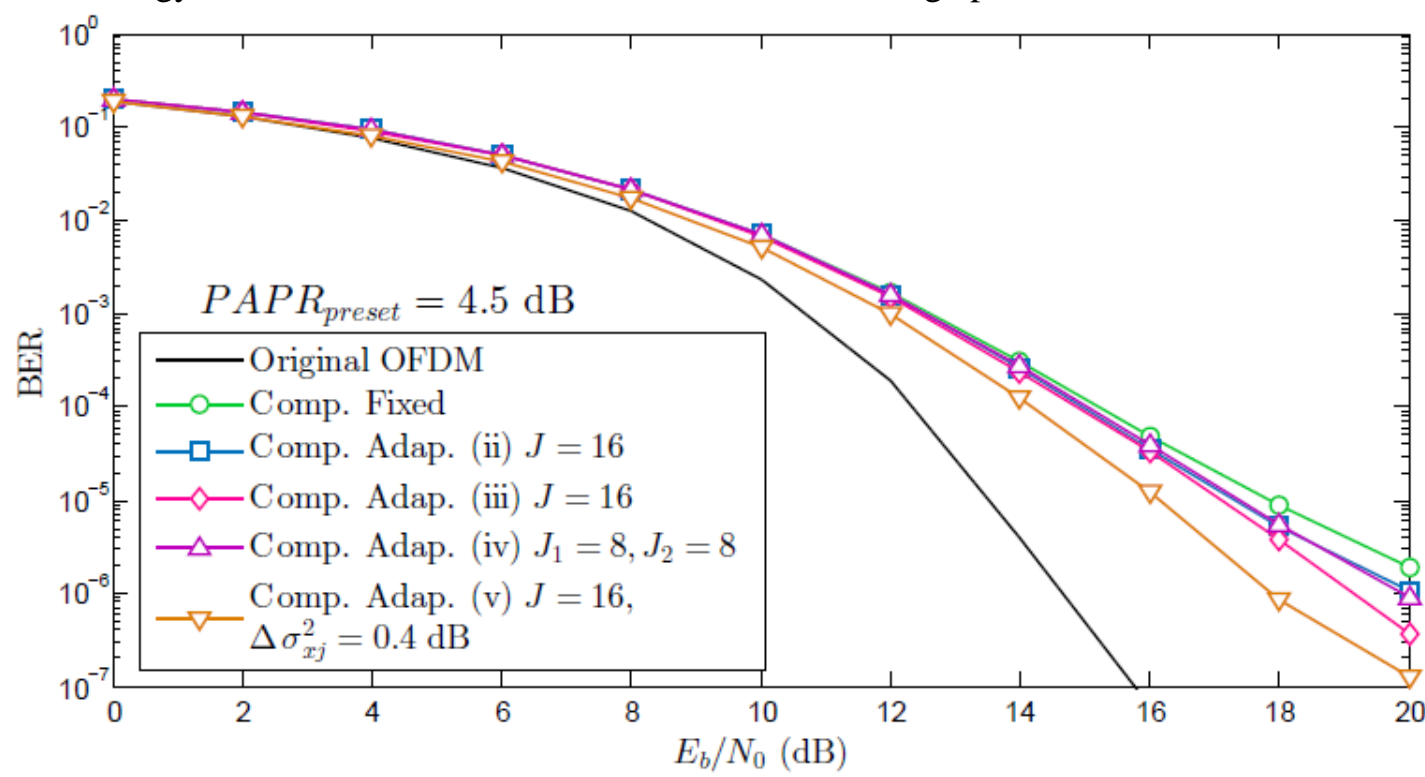

Fig. 11. BER performance of original OFDM signal and OFDM signal transformed with fixed and adaptive companders in scenarios (ii)-(v), over AWGN channel, using 16-QAM modulation.

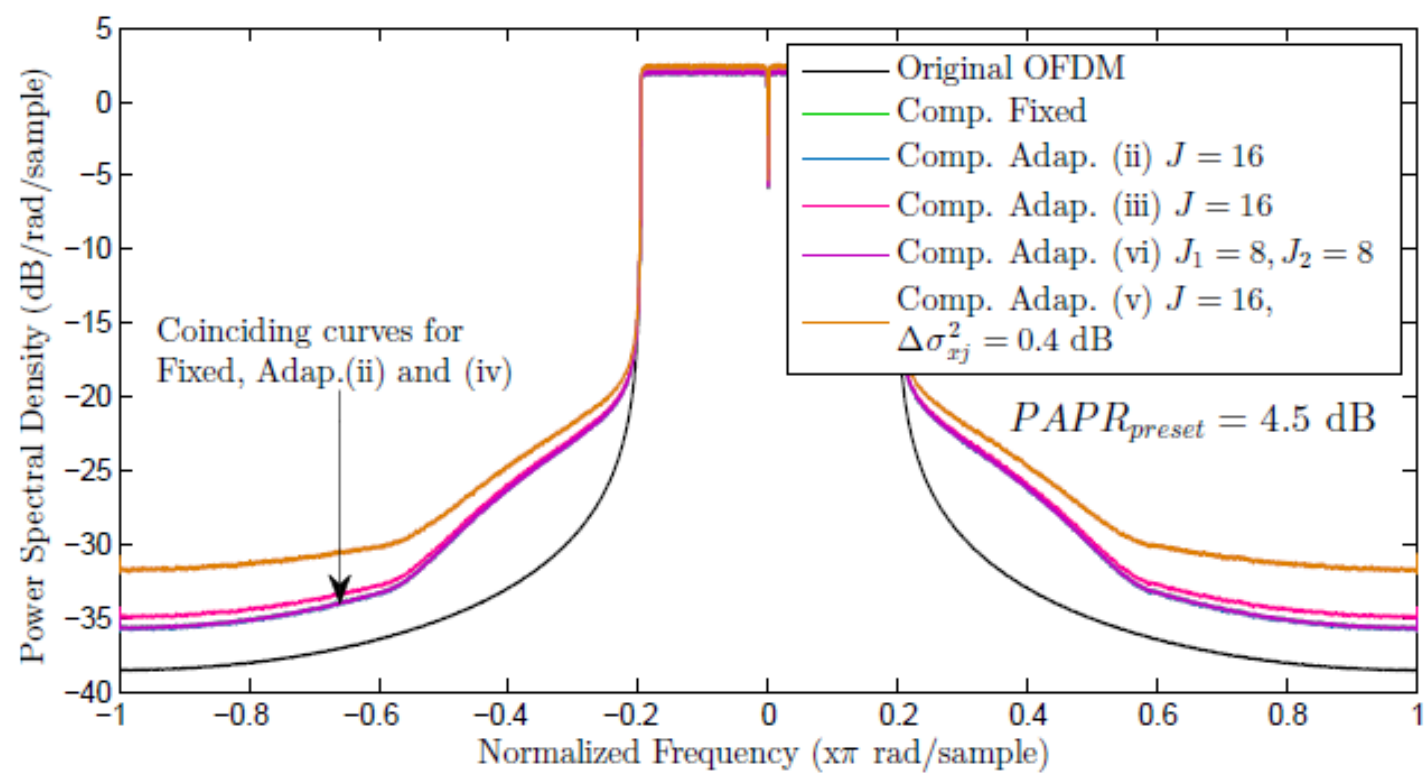

Fig. 12. PSDs of original OFDM signal and OFDM signal transformed with fixed and adaptive companders in scenarios (ii)-(v). OFDM signals are based on 16-QAM constellation.

Moreover, the slope $k$ remains same for all subsets when clipping level is changed according to average power. So the number of parameters needed to be stored in memory remains same in scenarios (i), (ii) and (v).

The results presented clearly demonstrate that with the proposed scheme, it is possible to cover a wide range of operating conditions for the system with reduced PAPR without introducing added distortion; a feat not achievable with deterministic companders. 
The trade-offs are shifted to added computational complexity required for calculating the input feature, additional memory required to store parameter values and requirement of side information for decompanding.

\subsection{Complexity Analysis}

For the two features used in the simulations, computational complexity is given below. Amplitude calculations and companding function arithmetic are common in fixed and adaptive companders, for which complexity analysis and comparison is presented in [3]. Approximately 1.32NL floating point multiplications and additions are required for fixed companding with $P A P R_{\text {preset }}=4.5 \mathrm{~dB}$ [3]. Additional complexity at the transmitter, involved in introducing adaptive behavior in the proposed scheme, is given as follows:

- For feature in Eq. (15), $S_{1}=$ Power $_{\text {avg }}=\frac{1}{N L} \sum_{n=0}^{N L-1}\left|X_{k}\right|^{2}$ : Average power of the input symbol can be calculated with low complexity from the frequency domain representation using QAM values. Out of $N L$ values in the sum, only $N_{\text {data }}$ are data bearing subcarriers while rest are pilot and null sub-carriers. Hence $N_{\text {data }}$ (192 in Fixed WiMAX) floating point additions and one multiplication per symbol will be required if symbol to energy mappings for the constellation are pre-calculated as a look-up table (LUT).

- For feature in Eq. (17), $S_{2}=\sum_{A \in\left\{\left|x_{n}\right|\right\}, A>\alpha_{c}}\left(A^{2}-\alpha_{c}^{2}\right):$ On average, $N L \int_{\alpha_{c}}^{\infty} f_{A}(x) d x\left(0.06 N L\right.$ for $\left.P A P R_{\text {preset }}=4.5 d B\right)$ floating point multiplications and additions are required per symbol.

Additionally, $J$ comparisons per symbol will be required to enable the adaptive compander select its parameters according to the value of calculated feature. In Table 1, $\mathrm{C}\left(\mathrm{S}_{1}\right)$ and $\mathrm{C}\left(\mathrm{S}_{2}\right)$ denote complexities of $\mathrm{S}_{1}$ and $\mathrm{S}_{2}$, respectively, and represents the required number of arithmetic operations as given above.

A summary of changes observed in the performance metrics is given in Table 1 . The highlighted cells show the aspects in which the performance differs from the corresponding fixed compander for each of the cases considered in simulations. For a given value of $P A P R_{\text {preset }}=4.5 \mathrm{~dB}$, trade-offs in respective cases are possible among the performance measures highlighted in each column.

Table 1. Summary of performance metrics for the proposed companding scheme

\begin{tabular}{|l|c|c|c|c|c|c|}
\hline & 4-QAM & \multicolumn{5}{|c|}{ 16-QAM } \\
\cline { 3 - 7 } & & (i) & (ii) & (iii) & (iv) & (v) \\
\hline $\begin{array}{l}\text { Best PAPR at } \\
\text { CCDF }=10^{-4}\end{array}$ & $4.55 \mathrm{~dB}$ & $4.8 \mathrm{~dB}$ & $4.8 \mathrm{~dB}$ & $4.8 \mathrm{~dB}$ & $4.67 \mathrm{~dB}$ & $4.8 \mathrm{~dB}$ \\
\hline Bit error rate & unchanged & unchanged & reduced & reduced & reduced & reduced \\
\hline Side-lobe level & unchanged & unchanged & unchanged & increased & unchanged & increased \\
\hline $\begin{array}{l}\text { Side } \\
\text { information }\end{array}$ & $\left\lceil\log _{2} J\right\rceil$ & $\left\lceil\log _{2} J\right\rceil$ & $\left\lceil\log _{2} J\right\rceil$ & $\left\lceil\log _{2} J\right\rceil$ & $\begin{array}{c}\left\lceil\log _{2} J\right], \\
J=J_{1} J_{2}\end{array}$ & $\left\lceil\log _{2} J\right\rceil$ \\
\hline $\begin{array}{l}\text { Computational } \\
\text { complexity }\end{array}$ & $\mathrm{C}\left(S_{2}\right)$ & $\mathrm{C}\left(S_{1}\right)$ & $\mathrm{C}\left(S_{1}\right)$ & $\mathrm{C}\left(S_{1}\right)$ & $\begin{array}{l}\mathrm{C}\left(S_{1}\right)+ \\
\mathrm{C}\left(S_{2}\right)\end{array}$ & $\mathrm{C}\left(S_{1}\right)$ \\
\hline $\begin{array}{l}\text { Memory } \\
\text { requirement }\end{array}$ & $2 J+1$ & $2 J+1$ & $2 J+1$ & $2 J+2$ & $J_{1}+2 J_{1} J_{2}$ & $2 J+1$ \\
\hline $\begin{array}{l}\text { Average } \\
\text { power }\end{array}$ & unchanged & unchanged & unchanged & increased & unchanged & increased \\
\hline
\end{tabular}




\section{Conclusion}

In this paper, a new adaptive companding scheme is proposed in which the compander selects its parameters from a pre-determined set according to certain statistical features of the input symbols. This is shown to mitigate the degrading effects of deviation of amplitude statistics from Rayleigh distribution, which is significant in case of larger QAM constellations. Simulation results show that the employed strategy improves the overall performance of the system by bringing the compander output signal attributes closer to optimal. The scheme also enhances the system's adaptability to input and channel conditions by facilitating more evenly distributed trade-offs as compared to those possible with existing PAPR reduction schemes.

\section{References}

[1] T. Jiang and Y. Wu, “An overview: Peak-to-average power ratio reduction techniques for OFDM signals,” IEEE Transactions on Broadcasting, vol. 54, no. 2, pp. 257-268, Jun. 2008. Article (CrossRef Link)

[2] Y. Rahmatallah and S. Mohan, "Peak-to-average power ratio reduction in OFDM systems: A survey and taxonomy,” IEEE Communications Surveys \& Tutorials, vol. 15, no. 4, pp. 1567-1592, Nov. 2013. Article (CrossRef Link)

[3] M. Hu, Y. Li, W. Wang, and H. Zhang, "A piecewise linear companding transform for PAPR reduction of OFDM signals with companding distortion mitigation," IEEE Transactions on Broadcasting, vol. 60, no. 3, pp. 532-539, Sep. 2014. Article (CrossRef Link)

[4] P. Yang and A. Hu, "Two-piecewise companding transform for PAPR reduction of OFDM signals,” in Proc. of Wireless Communication and Mobile Computing Conference (IWCMC), Istanbul, Turkey, pp. 619-623, Jul. 2011. Article (CrossRef Link)

[5] T. Jiang, Y. Yang, and Y.-H. Song, "Exponential companding technique for PAPR reduction in OFDM systems,” IEEE Transactions on Broadcasting, vol. 51, no. 2, pp. 244-248, Jun. 2005. Article (CrossRef Link)

[6] S.-S. Jeng and J.-M. Chen, "Efficient PAPR reduction in OFDM systems based on a companding technique with trapezium distribution,” IEEE Transactions on Broadcasting, vol. 57, no. 2, pp. 291-298, Jun. 2011. Article (CrossRef Link)

[7] Y. Wang, L.-H. Wang, J.-H. Ge, and B. Ai, “An efficient nonlinear companding transform for reducing PAPR of OFDM signals,” IEEE Transactions on Broadcasting, vol. 58, no. 4, pp. 677684, Dec. 2012. Article (CrossRef Link)

[8] J. Hou, J. Ge, D. Zhai, and J. Li, "Peak-to-average power ratio reduction of OFDM signals with nonlinear companding scheme,” IEEE Transactions on Broadcasting, vol. 56, no. 2, pp. 258-262, Jun. 2010. Article (CrossRef Link)

[9] S. P. DelMarco, "General closed-form family of companders for PAPR reduction in OFDM signals using amplitude distribution modification,” IEEE Transactions on Broadcasting, vol. 60, no. 1, pp. 102-109, Mar. 2014. Article (CrossRef Link) 

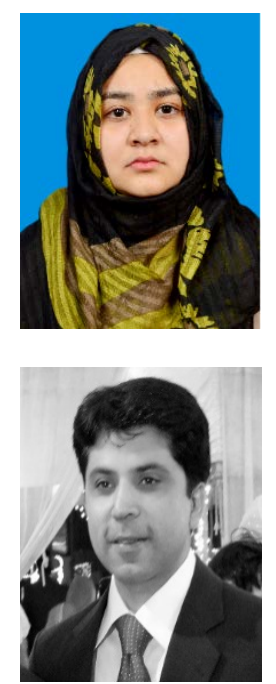

Sana Mazahir received her Bachelors in Electrical Engineering from College of Electrical and Mechanical Engineering, National University of Sciences and Technology (NUST), Rawalpindi, Pakistan, in 2013, where she is currently pursuing Masters in Electrical Engineering (DSP and Communication Systems). She is also working as graduate research assistant in System Analysis and Verification (SAVe) lab at NUST. Her research interests include signal processing for wireless communications, probabilistic analysis and modeling of digital systems and approximate computing.

Shahzad Amin Sheikh received the B.S. degree in Electrical Engineering from Eastern Mediterranean University (EMU), North Cyprus, in 1995 and Masters in Electrical Engineering from University of New South Wales (UNSW), Sydney, Australia, in 2002. In 2008 he received the Ph.D. from Southwest Jiaotong University (SWJTU), Chengdu, P. R. of China in the field of Information \& Communication Systems Engineering. Currently, he is working as a faculty member in Electrical Engineering Department at College of E \& ME (a constituent college of National University of Sciences \& Technology (NUST), Rawalpindi, Pakistan). His research interests are blind channel equalization and estimation, DSP and its applications in digital communication and biomedical engineering. 